\title{
Methodology of teaching literature with digital means
}

\section{[La methodologie de l'enseignement de la litterature avec le numerique]}

\author{
Valbona Gashi-Berisha - Nerimane Kamberi
}

DOI: 10.18355/XL.2019.12.01XL.11

\begin{abstract}
Education in general adapts according to the evolution of our societies and technological evolution. The textbook has long reigned in all educational systems in all countries. Today, thanks to developments in technology, the teaching and learning of literature have new teaching aids. Changes as revolutionary as the digital world offer a transformation in education by integrating the text-image-screen relation to a reflection of the teaching of literature more based on the supports (computer, tablets, connected objects) and their tools (sites, software, web).

Integrating technologies into the teaching process encourages teachers and learners to use information and communication technologies (ICTs). For the past few years, digital technology has been providing tools (Dropbox, Annotation Studio, PairForm ...) that make it possible to organize and share documents and pedagogical files between teachers and students. This new digital strategy is also an integral part of the teaching of literature. We can then wonder about this new form of teaching: how does the methodology of digital technology transform the process of teaching and learning of literature?
\end{abstract}

Key words: Teaching, platform, literature, supports, tools

\section{Résumé}

L'enseignement en général s'adapte en fonctions de l'évolution de nos sociétés et de l'évolution technologique. Le manuel scolaire a longtemps régné dans tous les systèmes éducatifs. Aujourd'hui, grâce aux développements de la technologie, l'enseignement et l'apprentissage de la littérature possèdent de nouveaux supports pédagogiques. Des changements aussi révolutionnaires que l'univers numérique offrent une transformation dans l'éducation en intégrant la relation texte-image-écrans à une réflexion de l'enseignement de la littérature davantage appuyé sur les supports (ordinateur, tablettes, objets connectés) et leurs outils (sites, logiciels, web).

L'intégration des technologies dans le processus d'enseignement encourage enseignants et apprenants à utiliser les technologies de l'information et de la communication (TIC). Le numérique met à disposition, depuis quelques années, des outils (Dropbox, Annotation Studio, PairForm...) qui permettent d'organiser et de partager des documents et des dossiers pédagogiques entre enseignants et étudiants. Cette nouvelle stratégie du numérique fait également partie intégrante de l'enseignement de la littérature. On peut alors s'interroger sur cette nouvelle forme d'enseignement : en quoi la méthodologie de la technologie numérique transforme l'enseignement et l'apprentissage de la littérature ?

Mots clés : enseignement, plateforme, littérature, supports, outils

\section{Introduction}

Dans cet article, nous allons élaborer la méthodologie de l'enseignement avec le numérique, l'introduction du numérique et les démarches effectuées à l'organisation des cours et des formations. L'exploitation des textes numériques et la lecture sur des formats électroniques transforment complètement le système d'enseignement. Depuis l'apparition des outils informatiques, des cours en direct étaient enregistrés et ensuite

XLinguae, Volume 12 Issue 1XL, January 2019, ISSN 1337-8384, eISSN 2453-711X 
diffusés au public. Le système éducatif a considéré ces innovations et cette réussite comme des enjeux essentiels. L'Université de la Sorbonne, dans le cadre d'émissions radiophoniques émises par Radio Sorbonne, a diffusé des cours dès 1947. Plus tard, pendant les années 1997 et 2002, le projet «Encyclopédie sonore : j'écoute les universités ", développé par plusieurs universités françaises et étrangères, a permis la mise en ligne de près de 7000 enregistrements de conférences et de cours regroupant 347 disciplines. L'Encyclopédie Sonore est un service éducatif en ligne... de données documentaires et d'un système de diffusion de conférences universitaires. Encyclopédie Sonore, 2002, en ligne.

L'un des exemples les plus connus est sans doute l'enregistrement des cours donnés par le philosophe Gilles Deleuze à l'Université de Paris 8 entre 1979 et 1987 et mis à disposition depuis au format numérique sur le site de l'Université (Astier, 2010). Université de Paris 8, 2001, en ligne.

Des cours en direct ont été enregistrés et diffusés à la télévision aussi par le Conservatoire national des arts et métiers (CNAM), dès 1963. Il continue toujours de diffuser des cours et offre :

- la formation professionnelle supérieure tout au long de la vie,

- la recherche technologique et l'innovation,

- la diffusion de la culture scientifique et technique. (CNAM)

\section{Formation : espace numérique}

Plusieurs établissements universitaires ont élaboré des projets dans la perspective de comprendre l'impact sur le lien entre formation des enseignants et pédagogie universitaire numérique. Durant plusieurs années, dans beaucoup de pays sont organisées des formations. En France, la formation Pairform@nce concerne les enseignants du premier et du second degré et FoDESIT visait la formation d'enseignants du supérieur (de 2002 à 2007).

Le Conservatoire national des arts et métiers (CNAM) est le premier opérateur public de formation professionnelle supérieure tout au long de la vie :

Il s'est aujourd'hui fait le pionnier de la formation par le numérique, en proposant par exemple une offre de cours accessibles à distance (Foad), lui permettant de toucher tous les publics, y compris les plus isolés. (CNAM, 2016).

Entre 2013 et 2016, le Conservatoire a produit 21 MOOC (Massive Open Online Courses ou cours en ligne ouverts et massifs) qui connaissent un succès croissant. Avec 350000 inscriptions sur la plateforme France université numérique (FUN). Il lance, au niveau national en février 2016, l'ouverture de la nouvelle plateforme pédagogique Moodle, la plateforme d'enseignement à distance Moodle, composante du nouvel espace numérique de formation (ENF), qui a pour ambition de faciliter les interactions entre enseignants et élèves, et d'accompagner au mieux l'évolution des pratiques pédagogiques, tout en permettant de développer les collaborations avec d'autres acteurs de l'enseignement supérieur

Le nouveau modèle éducatif a pris de larges dimensions en évoquant aussi l'évaluation par le numérique. Dans son rapport parlementaire de 24 février 2012, Jean-Michel Fourgous, député des Yvelines, écrit sur l'innovation des pratiques pédagogiques par le numérique et la formation des enseignants « Apprendre autrement » à l'ère numérique. Se former, collaborer, innover. Il cite que :

...les TICE ont un impact positif uniquement si elles sont employées lors de pratiques « nouvelles », elles-mêmes facilitées par l'utilisation des outils numériques : les TICE facilitent les apprentissages individualisés, collaboratifs, 
créatifs. Elles permettent de faire évoluer le système d'évaluation-sanction vers un système d'évaluation-accompagnement. (J.-M. Fourgous, 2018)

Des cours et des formations numériques de niveau universitaire sont nombreux. L'introduction du numérique a été engagée dès la fin des années 1990 avec le développement du e-learning et continue par la création des universités numériques thématiques (UNt) à partir de 2003. Ce phénomène s'élargit en 2010 avec l'apparition des MOOC, des cours à distance ouverts en ligne. Une grande diffusion des cours et des formations marque le succès de cette pédagogie numérique. C'est grâce à l'explosion et la dynamique autour des MOOC que la stratégie pédagogique fait du numérique un outil à part entière.

Des démarches éducatives à l'exploitation des tics ont été également organisées, en 2008 et en 2011, par l'UNESCO dans le but de former les enseignants pour qu'ils " soient capables d'aider les élèves à entrer dans une démarche d'apprentissage collaboratif, de créativité et de résolution de problèmes grâce à l'usage des TIC »(UNESCO, 2012). Ce Référentiel est articulé autour de trois approches de l'enseignement :

Dans la première approche, appelée Alphabétisation technologique, les élèves utilisent les TIC en vue d'un apprentissage plus efficace. Dans la seconde approche, Approfondissement des connaissances, les élèves acquièrent des connaissances approfondies dans les disciplines qu'ils étudient à l'école et les appliquent à résoudre des problèmes complexes et concrets. Dans la troisième approche, Création de connaissances, les élèves, citoyens et acteurs futurs de l'économie, créent les nouveaux savoirs indispensables pour bâtir des sociétés plus harmonieuses, enrichissantes et prospères. (Ibid., 2012).

Le projet du référentiel de l'UNESCO a pour objectif principal l'usage des TIC dans la réforme éducative. L'Institut français, de son côté organise tous les ans le festival international des cultures numériques appelé Novembre Numérique. Ce festival met en avant la création numérique sous toutes ses formes : production digitale, spectacle vivant, arts visuels, débat d'idées, éducatif et linguistique, cinéma, livre... Le programme est pluridisciplinaire et comprend quatre volets :

(De)Coder : Apprendre à utiliser les outils. C'est un programme sur l'initiation à la programmation informatique, comment faire une recherche sur le web et des plateformes de l'Institut français : IFprofs, Culturethèque.

- Jouer : Appliquer les formats participatifs et ludiques du numérique : création de jeu vidéo culturel, mashup cinéma, détournement et réappropriation. Utilisation du jeu (mindcraft, serious game) comme support de diffusion et de dialogue.

- Créer : Rencontrer les créateurs numériques, échanger entre entrepreneurs numériques des secteurs culturels, éducatifs, linguistiques, sociaux... Organiser des ateliers de créations d'objets connectés, de sites, de services u d'applications. Présenter au travers d'exposition le meilleur de la création française (transmédia, livre innovant, jeu vidéo, réalité virtuelle, performance...)

- Penser : Donner des clefs de compréhension de la société numérique : humanités numériques, open source, big data, démocratisation des 
savoirs, smart cities... Favoriser les formats innovants du numérique (hackathons, échanges participatifs, co-création) pour proposer d'autres formes de débat d'idées. (Institut français, 2017)

Cette approche à des fins d'apprentissage et de développement représente une intégration des nouvelles technologies dans le système éducatif. De ce point de vu, la maîtrise des outils numériques est très importante au développement du processus de l'apprentissage et à l'avenir de l'éduction en général.

\section{Les textes numériques}

Jules FERRY, dans sa circulaire aux recteurs du 7 octobre 1879 écrivait :

Vous parvenez peu à peu à faire abandonner par les intéressés les deux sortes de mauvais livres dont il faut que nos écoles se défassent : d'une part le livre vieilli... ; et d'autre part, le livre trop commode, où le maître trouve sa leçon toute faite, questions et réponses, devoirs et exercices, le livre qui dispense le maître d'expliquer et l'élève de comprendre, en substituant à l'imprévu de la classe parlée et vivante les recettes de l'enseignement systématique » (Borne, 1998).

De nos jours, cette classe parlée et vivante que Jules Ferry a mentionnée dans sa circulaire aux recteurs, voit le jour et peut s'appliquer en utilisant des logiciels et des tablettes numériques. Bien que la lecture sur écran représenterait une sorte de menace à l'encontre de la lecture du livre, le livre, considéré comme objet culte, a traversé des périodes et des générations et est devenu symbole du monde intellectuel. En ce sens, beaucoup de réflexions défendent l'idée que la lecture sur écran affaiblit la continuité intellectuelle du lecteur. D'ailleurs, cette menace est vue comme un processus de destruction surtout quand il s'applique à la lecture :

... comme le langage est, pour l'être humain, le premier véhicule de la pensée consciente, en particulier des formes supérieures de la pensée, les technologies qui le restructurent tendent à exercer une influence extrêmement forte sur notre vie intellectuelle (Carr, 2011).

L'utilisation des textes sur écrans, d'après Christian Vanderdope engendrerait une rupture avec le livre :

Alors que la lecture du livre est placée sous le signe de la durée et d'une certaine continuité, celle de l'hypertexte est caractérisée par un sentiment d'urgence, de discontinuité et de choix à effectuer constamment. En fait, chaque lien hypertextuel remet en question l'éphémère contrat de lecture passé avec le lecteur : celui-ci poursuivra-t-il sa quête en cliquant sur l'hypermot ou abandonnera-t-il ? (Vanderdope, 1999).

Si la lecture numérique ne cesse d'accroître sa facilité d'accès et d'utilisation, la lecture du livre imprimé continue à garder toujours un sentiment et un lien étroit avec le lecteur. En parallèle, la lecture du livre numérique diminue et dématérialise le livre traditionnel, considéré par le lecteur d'aujourd'hui comme un support physique qui ne séduit plus et qui révèle une sorte de difficulté à rivaliser avec le livre numérique. Par le biais de multiples outils, la lecture sur écran offre au lecteur des informations et des données innovantes facile à les procurer. En outre, l'accès illimité des textes en audiovisuels est un des avantages de ces nouveaux supports technologiques.

Les nombreuses études sur l'impact de la technologie dans le processus de l'apprentissage évoquent une évolution de documents pédagogique que les 
enseignants utilisent dans les classes. Mais qu'en est-il de l'usage du numérique dans l'enseignement/apprentissage de la littérature?

\section{La lecture avec le numérique}

La transformation du livre imprimé en livre numérique s'appuie sur la technique d'écriture et la réception des textes. L'apparition de l'écriture des hiéroglyphes, des manuscrits de papyrus et du passage de l'imprimé au numérique est un grand changement de la pensée humaine. Cette évolution de l'écriture a complètement changé la réception des textes et le mode de lecture.

À chaque fois, la culture écrite a conféré des rôles inédits aux objets et aux pratiques anciennes : des rôles inédits du rouleau à l'âge du codex, des rôles inédits de la communication et de la publication manuscrite à l'âge de l'imprimé. C'est à partir de cette perspective que l'on peut penser à la réorganisation de la culture de l'écrit que la révolution numérique impose (Chartier, 2012).

Cette transformation du livre en espace numérique est vue de la même manière par Michel Serres. Pour lui :

Il ne s'agit plus du même espace, évidemment, ni des mêmes rythmes et temps, ni des mêmes sons, ni de la même émotion, ni du même partage, ni du même continuum de style, ni du même récit, ni donc des mêmes amours, ni des mêmes âmes, ni peut-être des mêmes corps... (Serres, 2009).

L'accès à la lecture par les voies numériques sous-entend la modification en profondeur des pratiques et des méthodes de l'enseignement en général et de la littérature en particulier. Le recours à ce nouveau mode d'accès et d'exploitation des données textuelles se révèle particulièrement intéressant pour l'enseignant et l'apprenant. Les nombreux projets de recherche lancés depuis quelques années envisagent l'accès au texte par les voies numériques mais comme le constate Crinon, le changement dans l'enseignement demande plus d'effort :

Un immense chantier didactique est devant nous : concevoir des outils et des progressions, " de la maternelle à l'université », pour permettre à un plus grand nombre d'élèves d'accéder à la littératie étendue, en y incluant la littératie numérique (Crinon, 2012).

Les nouveaux supports pédagogiques évoluent et modifient le dispositif des enjeux de l'enseignement. De nos jours, une œuvre littéraire existe sous plusieurs formes: livre audio, adaptation filmique, vidéos de représentation si elle existe comme pièce de théâtre. La lecture scolaire des œuvres numériques contemporaines pour Saemmer est multidisciplinaire :

Grâce à son caractère multidisciplinaire, une lecture analytique d'œuvres automatiquement générées, animées ou hypermédiatiques peut également trouver sa place dans différents contextes pédagogiques (Saemmer, 2010).

L'évolution de la lecture trouve une forme d'extension dans d'autres supports pédagogiques, un changement de mode langagier de l'œuvre. De plus, les enseignants considèrent les documents autour du texte comme des " aides » pour " encadrer l'étude des textes », ce qui conduit au développement de pratiques de contextualisation. La manière même de lire et de penser a évolué avec l'arrivée des hyperliens (Fenniche 2011) dans un hypertexte, il est facile de changer de site sans 
s'en apercevoir. Avec le web 2.0, les frontières entre "producteurs " et « consommateur » sont brouillées, rendant difficile la détermination du réel auteur. Il n'y a pas de frontière physique comme l'est la reliure d'un livre. Cette absence de limite géographique peut modifier en profondeur le sens de ce qui est lu : en suivant les hyperliens, on intègre, au texte initial, de nouveaux contextes, lesquels changent sa logique et sa compréhension. La lecture demeure une priorité pour tous les systèmes éducatifs visant la capacité à lire et à comprendre des textes des plus simples aux textes des plus difficiles. Ce qui est important dans cette tendance de lecture, c'est le développement de l'enseignement sur plusieurs supports, que Crinon (2012), Lebrun et Lacelle (2012), nomment " séance composite » ou " multimodale ». Celle-ci implique de nouvelles compétences professionnelles, mais suscite également de nouvelles difficultés dans le processus pédagogique. Le lecteur d'aujourd'hui doit avoir des connaissances de ces nouveaux « espaces de lecture », doit être capable de chercher et de trouver toutes les informations dont il a besoin. Il est impératif de savoir repérer la source et l'auteur afin de juger de la crédibilité de l'information trouvée.

\section{La lecture multimodale}

Il existe plusieurs formats pour lire des e-books ou livres numériques. Un e-book ou livre numérique est un livre édité et diffusé en version numérique. Il est disponible sous forme de fichier, qui peut être téléchargé, stocké et lu sur différents supports de lecture numérique tel que : liseuse, smartphone, tablette ou ordinateur.

Selon la définition de Wikipédia le PDF (Le Portable Document Format) est un langage de description de page présenté par la société Adobe System en 1992 et qui est devenu une norme ISO en 2008. L'avantage de ce format PDF comme fichier informatique, est qu'il préserve les polices de caractères, les images, les objets graphiques et la mise en forme de tout document source, quelles que soient l'application et la plate-forme utilisées pour le lire. Mais le format PDF n'est pas adapté aux outils de lecture des smartphones ou des liseuses. (Https://fr.wikipedia.org/)

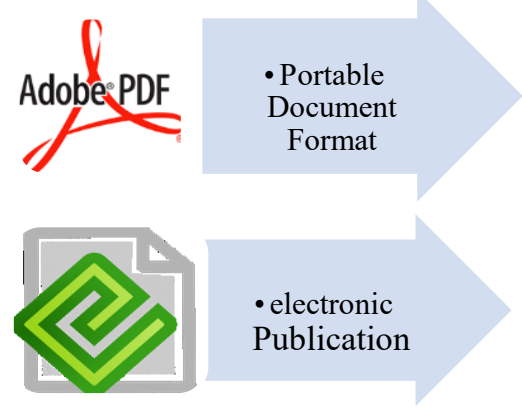

Figure 1. Lecture multimodale

\section{La lecture au format EPUB}

L'EPUB est conçu pour faciliter la mise en page du contenu, le texte affiché étant ajusté au tout dernier moment en fonction du dispositif d'affichage et des choix ergonomiques du lecteur au moment précis de la lecture, choix totalement différent de celui du format PDF (Wikipédia, EPUB).

À l'origine appelé Open e-book, ce format a pris le nom d'EPUB à partir de sa version 2. L'EPUB est un format de production, d'échange et de lecture pour les livres numériques, basé sur des standards web. Le contenu structuré est interprété 
pour une mise en forme adaptée à la lecture, quelle que soit la taille de l'écran. Au fil des versions de la norme, l'EPUB permet, hormis des contenus sémantiques simples (textes, images), l'intégration et l'interprétation de contenus enrichis (dont XHTML, CSS, SVG, images, etc.) Il s'agit de la dernière version de la norme EPUB rendue publique par l'IDPF en octobre 2011. La norme intègre des fonctionnalités supplémentaires permettant davantage d'interactivité et de scénarisation. Performant sur le confort de lecture, l'ePub3 permet l'intégration de vidéos, de fonctions interactives, d'animations et de sons. Le format EPUB contient plusieurs caractéristiques : il est de pouvoir contenir tous les éléments typiques d'un document sur le web... Les documents contenus dans un fichier EPUB sont rangés dans les dossiers pour les chapitres (texte), les images, le son (audio) et les films (vidéo) (Marcoux, 2014).

Un nouveau format EPUB 4 par Readium permet la lecture sur tous les supports : liseuses, tablettes, smartphones, ordinateurs. L'association créatrice du format de livre numérique EPUB, International Digital Publishing Forum l'IDPF a installé son siège à Paris en 2015, ainsi l'EDRLab, European Digital Reading Lab, devient l'un des centres de la réflexion pour l'avenir de la lecture numérique. Depuis la création, ce laboratoire s'investit à améliorer la lecture numérique en open source à l'échelle européenne, à travers le développement de logiciels et de standards (EPUB). Aujourd'hui, une quarantaine d'applications de lecture utilisent Readium.

\section{Les bibliothèques numériques}

Les livres numériques jusqu'en 2014 représentaient une part relativement faible dans le marché du livre en France, estimée à 4,1\% par le SNE. On compte aujourd'hui environ 120000 livres numériques français sous droit disponibles à la vente, répartis entre nouveautés et catalogues de fonds. Selon le SNE, 6 éditeurs sur 10 proposent une offre numérique. Pour améliorer cet état de fait, le Centre National du Livre attribue une aide (sous forme de subvention) afin de soutenir les éditeurs dans la numérisation rétrospective de leurs fonds et la diffusion numérique de documents sous droits, en lien avec Gallica. La numérisation des œuvres indisponibles dans le cadre du projet ReLIRE (registre des livres indisponibles en réédition, projet mené par la $\mathrm{BnF}$ ) rendra également disponibles des dizaines de milliers d'ouvrages du patrimoine littéraire.

Gallica est la bibliothèque numérique de la Bibliothèque nationale de France et de ses partenaires. En ligne depuis 1997, elle s'enrichit chaque semaine de milliers de nouveautés et offre aujourd'hui accès à plusieurs millions de documents. Elle permet d'effectuer des recherches au sein de l'ensemble des fonds numérisés. Chaque document peut être téléchargé intégralement ou partiellement : l'internaute peut facilement construire et enrichir sa propre bibliothèque.

La Bibliothèque nationale de France BNF à travers Gallica propose des dossiers pédagogiques autour d'une œuvre ou d'un auteur, un ensemble de textes et de documents de référence issus des collections de la Bibliothèque. Gallica est l'une des plus importantes bibliothèques numériques accessibles gratuitement sur l'internet. Elle offre l'accès à tous types de documents : imprimés (livres, presse et revues) en mode image et en mode texte, manuscrits, documents sonores, documents iconographiques, cartes et plans. Aujourd'hui Gallica compte 4988 777documents en ligne (BNF, Gallica, en ligne).

Le site pédagogique de la Bibliothèque nationale de France met en ligne des ressources à travers du portail « classes » disponible dans http://classes.bnf.fr/. Ce site offre la possibilité de conduire des recherches dans toutes les ressources et dans toutes les images : L'offre pédagogique en ligne représente plus de 85000 pages de dossiers, 65000 images commentées, des albums iconographiques thématiques, des documents à explorer de manière interactive, des documents audiovisuels, des pistes 
pédagogiques, des ateliers et des jeux, des chronologies, des bibliographies, des fiches pédagogiques téléchargeables, des recherches guidées dans Gallica, etc.

L'édition enrichie met en ligne la dernière nouveauté $A u$ Bonheur des Dames. C'est une approche à la fois ludique et scientifique pour que les enseignants et les étudiants puissent découvrir l'œuvre majeure d'Émile Zola. Elle offre de nombreux enrichissements autour du texte, lecture d'extraits par Lambert Wilson, notes explicatives, documents des collections $\mathrm{BnF}$, accès au dossier préparatoire de l'auteur.

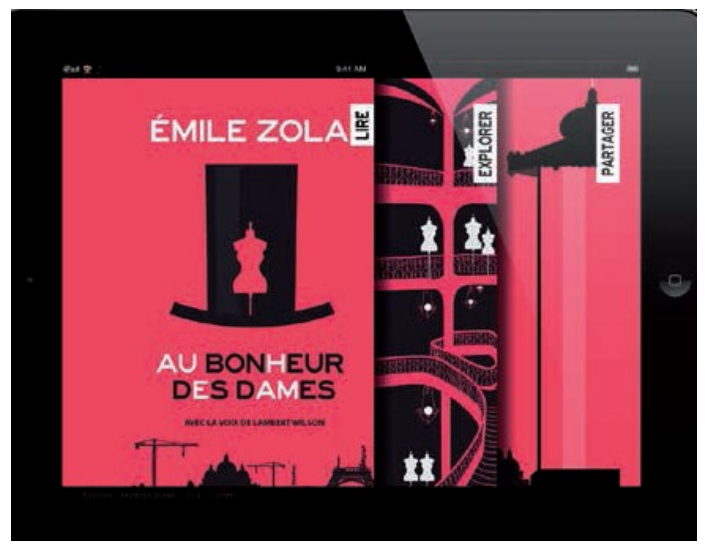

Source : http://bonheurdesdames.bnf.fr

L'édition enrichie $A u$ Bonheur des Dames comprend deux parties: lire et explorer. Lire- est un volet centré sur la lecture et la découverte du texte. Le lecteur peut choisir d'afficher une sélection de pages du manuscrit de Zola. En même temps, il peut découvrir des extraits à travers de la voix de Lambert Wilson qui donne une interprétation vivante du texte. Le lecteur peut aussi accéder à un mode de lecture puisant aux documents ; notes, fiches pédagogiques, illustrations etc., Il peut consulter les feuillets manuscrits, les notes rédigées de l'écrivain et leur transcription qui enrichissent le dossier pédagogique.

Explorer - est un volet sous huit thèmes ;

1. L'impressionnisme

2. Les femmes

3. Zola écrivain

4. Le gout de l'Orient

5. Une société en mouvement

6. Le commerce

7. La révolution industrielle

8. Les transformations de Paris et l'architecture de fer

\section{Éduthèque : un service du numérique éducatif}

Ce service offre des ressources éducatifs dans l'espace numérique et des fiches pédagogiques sur les principales expositions. Comportant des analyses d'œuvres et de documents, elles peuvent être utilisées en dehors de l'exposition et servir de support à des cours. Certaines d'entre elles sont téléchargeables sur le site, les autres peuvent être envoyées sur simple demande : présentation des expositions virtuelles, expositions en affiches, parcours-découverte des expositions pour les élèves, dossiers pédagogiques sur cédérom.

La création des livres numériques enrichis par Gallica met des liens du texte avec l'image et des liens de l'hypertexte comme le son et la vidéo. Le service Éduthèque de Gallica met à la disposition des enseignants, sur inscription, son offre du portail « 
classes » avec la possibilité de télécharger plus de 20000 images en haute définition et 250 vidéos. Les ressources pédagogiques sont adaptées pour favoriser un apprentissage en groupe et permettre une réflexion afin de développer un esprit critique.

Les Essentiels de la littérature est un site qui présente les auteurs et les œuvres dans leur contexte historique et artistique. Il met en relation les textes avec les manuscrits, des corpus d'images et des entretiens audiovisuels. Anthologie, citations et extraits invitent à découvrir le texte intégral dans Gallica. En 2015, cette offre s'enrichit d'un site spécifiquement dédié à la littérature, avec un premier volet des « Essentiels " consacré à la littérature du XVIIIe siècle sous la direction de Michel Delon et en 2016 continue avec le deuxième volet sur la littérature du XIXe siècle sous la direction de José-Luis Diaz. (Gallica, Essentiels). Nous pouvons rechercher, sélectionner et regrouper, depuis le volet Dossiers d'écrivain, les dernières nouveautés qui enrichissent le dossier de support de l'enseignant pour un travail plus attractif et innovant.

\section{Dossiers d'écrivain}

Dans la page d'accueil de Gallica, on trouve les manuscrits de Flaubert, conservés à la Bibliothèque nationale de France et à la Bibliothèque municipale de Rouen. Ces manuscrits sont classés dans l'édition électronique sur le site web: «Les manuscrits de Flaubert », sous la direction de Y. Leclerc et D. Girard de l'Université de Rouen, 2009. [Ressource en ligne]

Nous allons parler des manuscrits sur Madame Bovary, réunis dans cette édition qui comprennent sept sections. Les folios numérisés et leur transcription sont consultables dans les sections Feuilleter et Consulter.

Feuilleter : l'édition matérielle. Ce mode de consultation permet de parcourir les différents manuscrits qui la composent (plan et scénarios, brouillons, manuscrit définitif autographe, manuscrit du copiste). L'affichage des folios à l'écran reproduit la présentation matérielle des folios dans les reliures des manuscrits conservés à la Bibliothèque de Rouen.

Consulter : l'édition génétique. Ce mode de consultation permet d'afficher pour chaque passage de l'œuvre l'ensemble des états rédactionnels du texte, depuis les premières ébauches jusqu'au texte publié.

Les tableaux génétiques : accès direct aux folios et aux transcriptions depuis un tableau de classement génétique. Cette interface a été conçue par Jean-Eudes Trouslard, à partir des conclusions et de la base de données mise en place par Marie Durel dans le cadre de son classement. Deux tableaux génétiques distincts sont proposés pour le classement des folios des plans et scénarios et les folios du roman. 

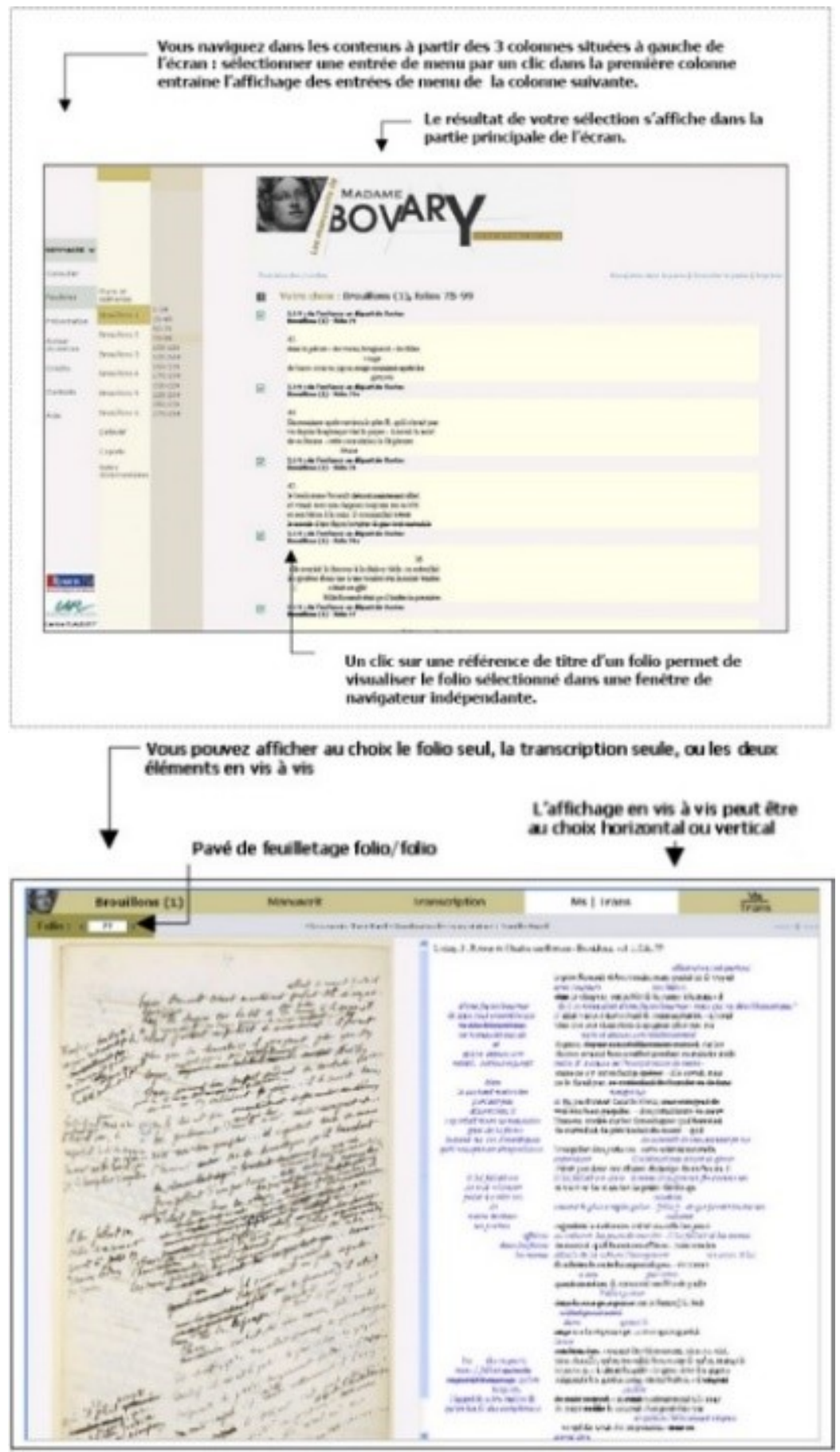

Tableau 1. Les folios en section consulter Tableau 2. Les transcriptions. Source : www.bovary.fr/ 
La Bibliothèque municipale de Rouen, depuis 1914 conserve tous les scénarios, brouillons et manuscrits de Madame Bovary de Gustave Flaubert, don effectué par Caroline Franklin Groult, nièce de Flaubert.

L'analyse génétique des manuscrits de Madame Bovary met d'ailleurs en évidence l'intensité des phénomènes de réécritures chez Flaubert: ont été relevé jusqu'à 50 folios rédigés pour parvenir au folio définitif (en l'occurrence, le folio 292).

Les codes des transcriptions sont :

- Le texte principal qui correspond au premier jet de Flaubert ou à la mise au propre du brouillon précédent est en caractères romains et en noir.

- Les ajouts marginaux ou interlinéaires sont en italiques bleus.

- Ce qui est barré est barré.

- Ce qui est souligné est souligné.

- Les passages biffés sont encadrés par une double accolade $\{\{\}\}$ et apparaissent à l'écran sur fond jaune.

- Les passages barrés d'une croix de Saint-André sont encadrés par un double crochet [[ ]].

- Les mots incertains sont suivis d'un astérisque. Deux astérisques encadrent une suite de mots incertains.

- $\quad$ Les mots ou les passages illisibles sont marqués [illis.]. On signale par des points de part et d'autre la longueur du texte non déchiffré.

\section{Description des manuscrits}

Les collections de littérature de la Bibliothèque nationale de France sont parmi les plus riches au monde. La littérature française est très largement représentée : manuscrits médiévaux, production éditoriale française moderne et contemporaine, littérature jeunesse, brouillons d'écrivains, épreuves originales... Ces ensembles exceptionnels, enrichis des collections des bibliothèques et institutions partenaires sont disponibles dans Gallica.

Avant la numérisation qui a permis la publication en ligne, le dossier des manuscrits de Madame Bovary a connu un important effort de reconditionnement, à la mesure du don fait à la Ville et sa Bibliothèque en 1914.L'état général des volumes de brouillons rendait la restauration de l'ensemble indispensable. Entre 1994 et 1999, la Direction du Livre et de la Lecture a financé donc le remontage de tous les feuillets dans une reliure de conservation, dont la réalisation a été confiée aux ateliers de restauration de la Bibliothèque nationale de France, rue de Richelieu. Chaque feuillet manuscrit est alors monté par une charnière sur un feuillet neutre d'un format plus grand que le papier du manuscrit lui-même. Cette présentation permet aujourd'hui de feuilleter l'œuvre sans avoir de contact avec le document original. Mais elle a également multiplié par deux le nombre de feuillets de chaque volume. L'objectif de la numérisation des manuscrits de Madame Bovary ne s'arrêtait pas à la production d'une nouvelle copie de substitution, mais constituait une simple étape dans la démarche générale de valorisation du projet, devant permettre ensuite la transcription intégrale des feuillets et leur classement informatisé.

La critique génétique se donne pour but l'étude des processus d'écriture par l'analyse des manuscrits. Elle a trouvé en Flaubert un terrain favorable à son développement. Flaubert gardait à peu près tout ce qui sortait de sa plume : "Jamais je ne jette aucun papier. C'est de ma part une manie ", écrit-il à Louise Colet le 26 avril 1853. À l'occasion, il peut montrer les preuves de son laborieux travail d'écriture : «Quand mon roman sera fini, dans un an, je t'apporterai mon manuscrit 
complet, par curiosité. Tu verras par quelle mécanique compliquée j'arrive à faire une phrase » (Bruneau, 1980, lettre du 15 avril 1852).

L'édition électronique de Madame Bovary est établie par Danielle Girard et Yvan Leclerc selon l'édition Charpentier de 1873. Les «tableaux génétiques » fondés sur le classement des brouillons sont effectué par Marie Durel et la modélisation de JeanEudes Trouslard, et permettent de naviguer dans l'édition. (Source https://www.bovary.fr. )

Cette édition permettra surtout aux enseignants de préparer des séquences pédagogiques utilisant l'ensemble des sources classées et bien organisées. Les brouillons de Madame Bovary sont donc présentés aujourd'hui sous la forme de 12 volumes, sans que la foliotation et la tomaison en soient changées : chaque tome est composé de deux parties séparées mais qui se suivent au niveau de la foliotation en conservant l'organisation qui leur avait été attribuée lors du premier regroupement :

Dans l'édition intégrale de Madame Bovary, il y a aussi les illustrations de A. Richemont, gravées à l'eau-forte par C. Chessa, Paris, F. Ferroud, 1905.

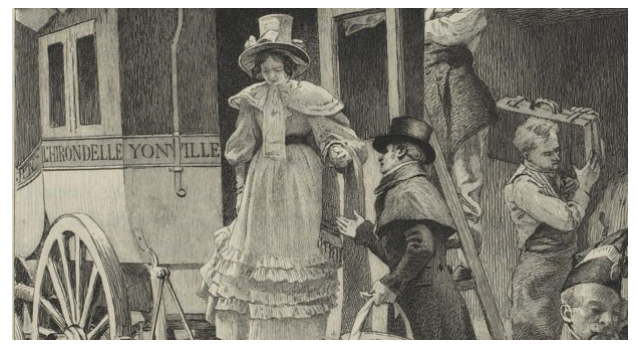

Image 1. - 2e partie, chapitre 14 : Rétablissement d'Emma (le voyage à Rouen). Source https://www.bovary.fr

La diversité des représentations auxquelles le roman de Madame Bovary a donné lieu permet aux étudiants de repérer des points communs et des divergences entre elles. Cette perspective comparatiste est stimulante pour comprendre les particularités et la complexité du roman. Flaubert était contre les illustrations de ses œuvres et voyait dans le rapport entre texte et images un appauvrissement de la pensée esthétique. Dans une lettre à Ernest Duplan, il exprimait son désaccord : « Jamais, moi vivant, on ne m'illustrera ». (Bruneau, Leclerc, 2007 : 221-222). Pour lui l'image, insérée dans le texte, bride l'imagination du lecteur : alors que son écriture cherche à susciter mille images, la gravure n'en fixe en définitive qu'une seule. Les images semblent aider la littérature de l'époque à se redéfinir et malgré cela l'auteur de Madame Bovary insiste et garde ses idées contre les illustrations. Après la mort de Flaubert, sa nièce Caroline Commanville, détentrice des droits littéraires, ne s'oppose plus à la réalisation de telles éditions.

Il faut admettre que pour les enseignants, l'œuvre littéraire gravée et illustrée est un support très important dans les cours de l'enseignement d'une langue étrangère. Le texte littéraire en tant qu'outil didactique nous permet d'explorer le pouvoir des mots utilisés par l'auteur et quand l'image y est présente c'est un renforcement et une sorte de facilité à l'analyse texte-image. L'image permet d'apporter un éclairage nouveau aux approches didactiques de la littérature en contexte universitaire sur les frontières entre l'illustration et la textualité. Les images dans les œuvres illustrées sur Madame Bovary peuvent aider les étudiants à mieux saisir le sens de l'histoire et les amener à interpréter le texte par rapport au visuel offert.

\section{Méthodologie - Recherche ciblée et échantillons}

Une enquête auprès de 55 étudiants a été menée à l'Université de Prishtina, notamment à la Faculté de philologie, au département de langue et de littérature 
françaises. La question suivante portait sur l'utilisation et l'exploitation des textes numériques et la lecture sur des formats électroniques. Les étudiants qui ont participé à cette enquête sont assidus aux cours et très motivés à l'utilisation des Tics. Le fait que le pourcentage d'étudiants de première année est plus élevé, c'est tout simplement parce qu'ils sont plus nombreux tandis que le pourcentage des étudiants de la deuxième année et de la troisième année, est tombé à $25 \%$. Voir le tableau 1 . L'âge des étudiants qui ont répondu à l'enquête est entre 18 et 20 ans (63\%), entre 21 et 25 ans $(31 \%)$ et seulement $6 \%$ entre 26 et 30 ans.

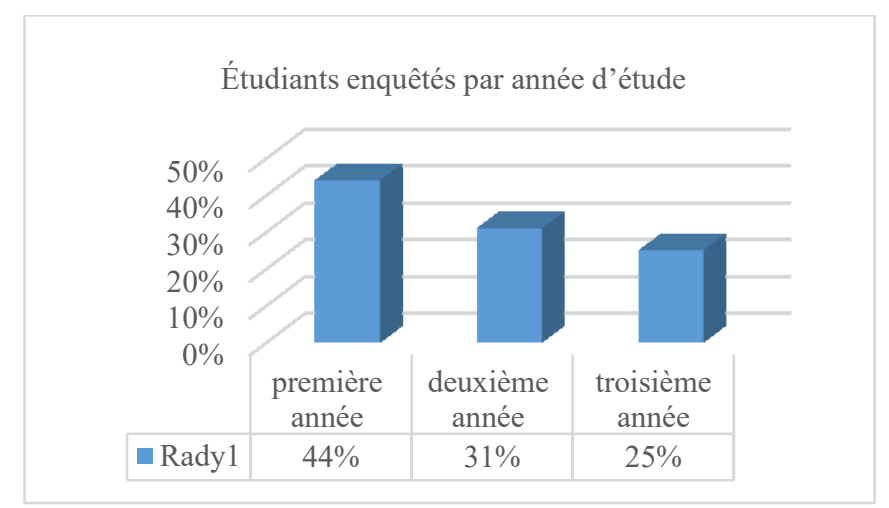

Tableau 1. Le pourcentage des étudiants enquêtés par année d'étude

\section{L'objectif de la recherche}

Le but de cette recherche est de voir les compétences des étudiants à l'utilisation des ressources numériques pour un meilleur encadrement pédagogique. Il est nécessaire de savoir la place qu'occupe aujourd'hui le numérique dans les pratiques de la lecture. Selon cette enquête, les étudiants du département sont bien intégrés à la transformation de la maîtrise de la lecture numérique. Le tableau 2 montre que les étudiants sont plus intéressés à la lecture du livre numérique soit $63 \%$ et un nombre moins important d'étudiants soit $37 \%$ qui continue à lire des livres imprimés.

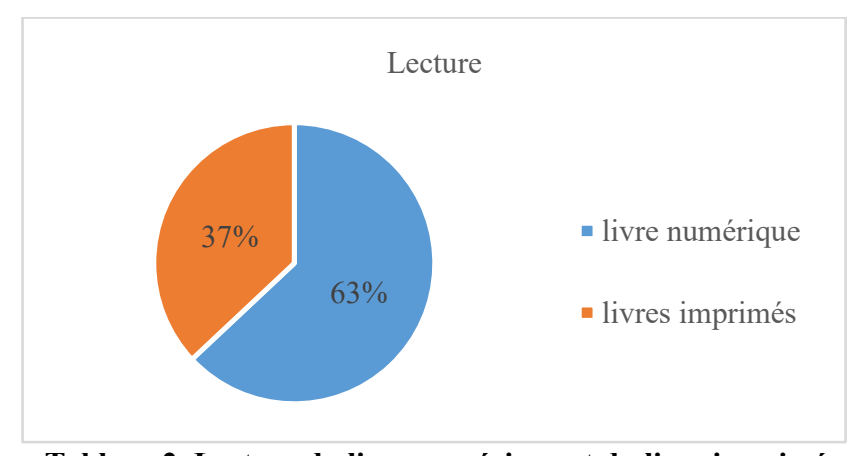

Tableau 2. Lecture du livre numérique et du livre imprimé

L'accès à l'information facile encourage plus en plus l'étudiant à lire et à faire des recherches sur internet. Il trouve que la lecture numérique est plus facile et plus attractives que la lecture classique avec des libres imprimés. Il faut noter que parmi les étudiants enquêtés, un petit nombre soit $15 \%$ des étudiants suivent déjà des cours de littérature et cinématographie et sont très intéressés à l'interactivité et l'usage des 
applications. Nous avons préparé, en juin 2018 un film intitulé OFFlife - L'addiction aux réseaux sociaux, et l'avons projeté au Centre Universitaire Francophone (CUF) de l'Université de Pristina. Le film traite une thématique très actuelle qui est la dépendance des réseaux sociaux par les jeunes, un phénomène qui envoie parfois des jeunes à la fatalité. Le film présente également la vie virtuelle des jeunes qui ont perdu leur sens de la vie réelle. Cela peut être lié à l'intérêt des étudiants à utiliser des logiciels pour monter le film et perfectionner l'usage des appareils électroniques. Parmi de nombreux logiciels qui sont en ligne gratuitement, nous avons choisi deux logiciels audio-visuels comme aide à la préparation des cours de littérature avec le numérique. Pour le montage du son, nous utilisons le logiciel Audacity et pour le montage vidéo nous avons choisi le logiciel Openshot.

\section{Conclusion}

Face aux nouveaux changements éducatifs et aux nouveaux modes de support, l'enseignement de la littérature avec le numérique s'impose progressivement. Les formats de mise en page des textes ont évolué de manière à rendre l'expérience de lecture la plus agréable possible. Ce changement de mode lecture vient grâce à l'univers numérique qui complète le livre imprimé en facilitant sa disponibilité et sa diffusion. C'est dans de pareilles réflexions que Jacques Michon, professeur de littérature à l'Université de Sherbrooke, cite :

Plutôt que de s'opposer à l'imprimé, l'écrit électronique le complète et le prolonge. Des textes anciens, qui ne sont plus disponibles en librairie ni même dans la majorité des bibliothèques publiques ou universitaires, refont maintenant surface dans la cybersphère. Le livre n'est plus un objet fermé sur lui-même, isolé dans son carcan typographique, mais grâce au numérique, il devient l'un des supports d'un vaste texte qui se déroule à l'infini. (Michon, 2008)

Le numérique permet avant tout de retrouver un nouvel élan dans le processus de l'enseignement et de l'apprentissage qui est avant tout une continuité pédagogique de l'enseignement classique vers l'enseignement numérique. Ce changement dans le processus de l'enseignement demande des multitâches pédagogiques de l'enseignant et de l'étudiant et des missions qui ont pour fonction le développement de l'acquisition des connaissances littéraires. La nouvelle technologie, utilisée dans le processus de l'enseignement littéraire détermine et oriente l'enseignant et l'étudiant vers le numérique répondant aux attentes du lecteur d'aujourd'hui grâce aux supports audiovisuels perçus comme plus attractifs qu'un simple livre imprimé. Le livre en version numérique ou le livre électronique est une réelle prise de conscience des enjeux littéraires permettant au lecteur de retrouver le goût et le plaisir de lire.

\section{Bibliographic references}

BORNE, D. 1998. Le manuel scolaire, Paris : La Documentation Française. En ligne. Available online: https://www.ladocumentationfrancaise.fr/var/storage/rapportspublics/994000490.pdf

BRUNEAU, J. - LECLERC, Y. 2007. Gustave Flaubert, Correspondance. Lettre a Georges Charpentier du 16 février 1879, volume V, Paris, Gallimard, « Bibliotheque de la Pleiade », 1973-2007, t. V. ISBN : 9782070116126.

BRUNEAU, J. 1980. Gustave Flaubert, Correspondance, tome II : Juillet 1851 Décembre 1858, Paris, Gallimard, «Bibliotheque de la Pleiade », 1973-2007, t. II. ISBN : 9782070106684

CARR, N. 2011. Internet rend-il bete ? Paris, Robert Laffont, ISBN 978-2221124437. CHARTIER, R. 2012. Qu'est-ce qu'un livre ? Metaphores anciennes, concepts des Lumieres et realites numeriques. Dans la revue Le Français aujourd'hui, L'enseignement des lettres et le numerique. 3 nr.178. ISBN : 9782200927820 
CNAM Conservatoire national des arts et metiers. 2016. En ligne. Available online : www.cnam.fr/infos-site.

CRINON, J. 2012. Enseigner le numerique, enseigner avec le numérique. Dans la revue Le Français aujourd'hui, L'enseignement des lettres et le numerique. 3 nr.178. pp.107-114. ISBN: 9782200927820

FENNICHE, R. 2011. Chapitre IV. Hyperlecture et culture du lien In : Lire dans un monde numérique [en ligne]. Villeurbanne: Presses de l'enssib, 2011 Available online :

http://books.openedition.org/pressesenssib/1092 ISBN : 9782375460481.

FOURGOUS, J.-M. Reussir l'ecole numerique, Rapport de mission parlementaire [En ligne] Available

online :

http://www.missionfourgoustice.fr/missionfourgous2/spip.php?article5

FOURGOUS, J.-M. 2012. Apprendre autrement a l'ere numérique Se former, collaborer, innover, En ligne. Available online :

https://www.ladocumentationfrancaise.fr/var/storage/rapports-publics/124000169.pdf

Institut français, Novembre numérique, le mois des cultures numériques, 2017.

GURA, R. - ROSTEKOVA, M. 2018. Francuzsky model profesionalizacie terciarneho vzdelavania na Slovensku. In Politicke vedy. [online]. Vol. 21, No. 3, 2018. ISSN 1335 - 2741, pp. 236-244. Consulté en mai 2018 : http://doi.org/10.24040/politickevedy.2018.21.3.236-244

Available online : www.institutfrancais.com/fr/prospective-numerique2017/novembre-numerique-le-mois-des-cultures-numeriques

HASKOVA, A. - MANDULAKOVA, S. - VAN MEROLDE,D. 2017. Problematic aspects of technology education in Slovakia. In: Communications - Scientific Letters of the University of Zilina. vol. 19, n. 1, pp. 75-80. ISSN 1335-4205.

LEBRUN, M. - LACELLE, N. - BOUTIN, J.-F. 2012. La littératie médiatique multimodale. De nouvelles approches en lecture-écriture à l'école et hors de l'école. Québec : PUQ. ISBN 978-2-7605-3470-4.

MARCOUX, F. 2014. "Le livrel et le format ePub". In: E. Sinatra Michael, VitaliRosati Marcello (edite par), Pratiques de l'édition numérique, collection «Parcours Numériques », Les Presses de l'Universite de Montreal, Montreal, pp. 177-189, ISBN : 978-2-7606-3202-8, en ligne, Available online :

www.parcoursnumeriques-pum.ca/le-livrel-et-le-format-epub

MICHON, J., 2008. La bataille de l'imprime a l'ere du papier electronique, in Le Ray Éric, Lafrance Jean-Paul (edite par), Le monde du livre a l'heure du numérique, en ligne, Available online: https://books.openedition.org/pum/16966 SAEMMER, A. 2010. Lire la litterature numerique a l'universite : deux situations pédagogiques. Ela. Études de linguistique appliquee, 160, (4), pp. 411-420. Available online: https://www.cairn.info/revue-ela-2010-4.htm-page-411.htm

SERRES, M. 2009. Recits d'humanisme, editions Le Pommier, Paris. ISBN 9782746504233.

UNESCO, un referentiel de competences Tice pour les enseignants, 2012, en ligne. Available online : https://unesdoc.unesco.org/ark:/48223/pf0000216910

Universite de Paris 8, La voix de Gilles Deleuze, 2001, en ligne, Available online : http://www2.univ-paris8.fr/deleuze.

Sitographie :

http://www.bovary.fr

http://gallica2.bnf.fr

http://gallica.bnf.fr/essentiels/

http://e-sonore.u-paris10.fr/

https://fr.wikipedia.org

XLinguae, Volume 12 Issue 1XL, January 2019, ISSN 1337-8384, eISSN 2453-711X 
Words: 6520

Characters: 43846 (24,36 standard pages)

Assoc. prof. dr. Valbona Gashi-Berisha

Department of French Language and literature

Faculty of Philology, University "Hasan Prishtina" of Prishtina

10000 Prishtina,

Kosovo

Valbonagashi@uni-pr.edu

Ass. Prof. dr. Nerimane Kamberi

Department of French Language and literature

Faculty of Philology, University "Hasan Prishtina" of Prishtina

10000 Prishtina,

Kosovo

nerimanekamberi8@gmail.com 